\title{
Controle Direto de Torque (DTC) com Estimação de Conjugado
}

\author{
Matheus A. G. Calsavara*. Lane M. R. Baccarini* \\ * Departamento de Engenharia Elétrica, Universidade Federal de São João Del Rei, \\ São João del-Rei, Minas Gerais, CEP: 36307-352 \\ (e-mail: matheus.guilarducci@hotmail.com,rabelo@ufsj.edu.br).
}

\begin{abstract}
The three-phase induction motors are widely used in industry once they can work in adverse environmental conditions due mainly to the inexistence of brushes. The speed control of these machines by electronic converters has been increasing in the last years, reducing the drives of loads by Machines of Continuous Current. However, in high-performance drives, speed control requires sophisticated control techniques. A technique adopted in industrial frequency converters is the Direct Torque Control (DTC) that aims to control torque and flux directly, instead of trying to control these variables indirectly. The use of a torquemeter to measure the torque, is onerous and decreases the robustness of the drive and also increases maintenance costs. Thus, the objective of this work is to propose a method of estimation of electromagnetic torque to be applied in the control loop to replace the torquemeter. Computational studies for motors of different characteristics and conditions of load operation were performed and show the robustness of the proposed method. The technique has strong industrial appeal because it requires only the signals of sensors present in the control loop.
\end{abstract}

Resumo: Os motores de indução trifásicos são amplamente utilizados na indústria, uma vez que podem trabalhar em condições ambientais adversas devido, principalmente, a inexistência de escovas. O controle de velocidade dessas máquinas por conversores eletrônicos vem crescendo nos últimos anos, reduzindo os acionamentos de cargas por Máquinas de Corrente Contínua. Entretanto, em acionamentos de alto desempenho, o controle de velocidade requer técnicas de controle sofisticadas. Uma técnica adotada em conversores de frequência industrial é o DTC (Controle Direto de Torque) que visa controlar o torque e o fluxo diretamente, em vez de tentar controlar estas variáveis indiretamente. A utilização de torquímetro para a medição do conjugado, onera e diminui a robustez do acionamento e aumenta também os custos de manutenção. Assim, o objetivo desse trabalho é propor um método de estimação de torque eletromagnético para ser aplicado na malha de controle em substituição ao torquímetro. Estudos computacionais para motores de diferentes características e condições de operação de carga foram realizados e mostram a robustez do método proposto. A técnica tem forte apelo industrial pois necessita apenas dos sinais de sensores presentes na malha de controle.

Keywords:DTC; Direct Torque Control; Torque Estimation; Applications in Industry; Frequency Converter

Palavras-chaves: DTC; Controle Direto de Torque; Estimação de conjugado; Aplicações na Indústria; Conversor de Frequência.

\section{INTRODUÇÃO}

Com o advento da eletrônica de potência foram surgindo diversas técnicas de controle de velocidade para motores de corrente alternada. Anteriormente, os motores de corrente contínua eram os mais utilizados para essa finalidade, mesmo diante do elevado custo de aquisição e manutenção. Entretanto, com o desenvolvimento do princípio por orientação de campo, apresentado em 1972 por Felix Blaschke, foi possível o desacoplamento dos controles de fluxo e torque, possibilitando a utilização dos motores de indução em aplicações que exigiam um alto desempenho dinâmico.

Dentre as diversas técnicas de controle vetorial, destaca-se o Controle Direto de Torque, ou simplesmente chamado de
DTC. Esse modelo apresentado por Takahashi em 1985 faz parte de um conjunto de técnicas de controle de torque aplicando princípios de orientação de campo para o estator e modulação por vetores espaciais. Apesar do intenso chaveamento do inversor de frequência e da necessidade de estimação de parâmetros como fluxo do estator e o torque eletromagnético da máquina, essa modalidade de controle é rápida e apresenta uma excelente regulação de velocidade quando implementada em malha fechada.

O DTC trabalha através de uma lógica responsável por fornecer os comandos de chaveamento para o inversor de frequência de acordo com três parâmetros: erro de torque, erro de fluxo e setor espacial onde se encontra o setor de fluxo. Ao todo são oito possíveis vetores de chaveamento, que vão sendo determinados de forma que a máquina mantenha a operação dentro dos limites especificados. 
Alguns artigos recentes sobre essa modalidade de controle apresentam evoluções para a técnica e desenvolvem alternativas para contornar algumas adversidades. Malla (2016) em um artigo publicado no IEEE analisa o emprego do DTC em conjunto com controladores que utilizam a lógica Fuzzy e sugere uma comparação e avaliação das variações do DTC-SVM. Além disso, trabalhos recentes também propõem aplicações práticas, como o uso do DTC para reduzir perdas nos motores de indução aplicados em veículos elétricos com alta vida útil de bateria (Tazerart, 2015). Tazerart (2015), em seu trabalho, buscou aproveitar as vantagens do DTC e do controle ótimo, desenvolvendo uma nova abordagem, denominada Controle de Torque Direto Ideal (ODTC).

Outro artigo recente também sugere o uso do Controle Direto de Torque em veículos elétricos. Ziane (2014) analisou a técnica acionando dois motores idênticos ligados em cada um dos eixos do veículo. No caso de funcionamento do modo 4WD (four wheel drive), os dois motores entram em operação para tracionar os eixos dianteiro e traseiro. Para o modo FWD (front wheel drive), o DTC opera em conjunto com apenas um motor, enquanto o eixo traseiro permanece sem aplicação de torque.

O objetivo principal desse trabalho é propor uma forma de estimar o torque eletromagnético para que possa ser utilizado pela lógica do DTC. A vantagem é a eliminação da necessidade da utilização de um sensor de medição de torque, que é altamente invasivo, além da redução do custo de manutenção e aquisição. Para isso foi utilizado um método apresentado por Lívia Mendes (Mendes, 2015) que utiliza as tensões e correntes de alimentação do motor. O método foi implementado em um sistema de malha fechada através de um controlador PID e de forma que as equações fossem referenciadas aos eixos dq.

\section{METODOLOGIA}

Os parâmetros do MIT de 3 HP utilizados nas simulações estão disponíveis em Krause et al. (2002). O modelo dinâmico da máquina foi implementado no software $\mathrm{MATLAB}^{\circledR}$. As equações diferenciais foram resolvidas pelo método de integração Runge Kutta de $4^{\mathrm{a}}$ ordem com um passo de integração igual a $10^{-5} \mathrm{~s}$.

As equações que descrevem o modelo dinâmico do Motor de Indução Trifásico são bastante conhecidas. Portanto, não serão descritas neste trabalho.

\subsection{Controle direto de Torque}

O Controle Direto de Torque (DTC - Direct Torque Control) foi apresentado por Takahashi (1985) e tornou-se a versão mais conhecida dentre um conjunto de técnicas vetoriais introduzidas na década de 1980. O DTC é uma técnica de controle baseada no chaveamento do inversor de frequência através da implementação de uma tabela lógica que dita a condição dos interruptores semicontrolados em função dos parâmetros de erro de torque, erro de fluxo do estator e setor espacial onde o fluxo do estator se encontra. A tabela lógica foi desenvolvida de forma que o torque e o fluxo no estator sejam controlados de forma direta, por isso, a cada instante de amostragem um novo vetor de chaveamento é escolhido.

Resumidamente, o esquema de funcionamento do DTC pode ser representado pelo diagrama de blocos da Figura 1:

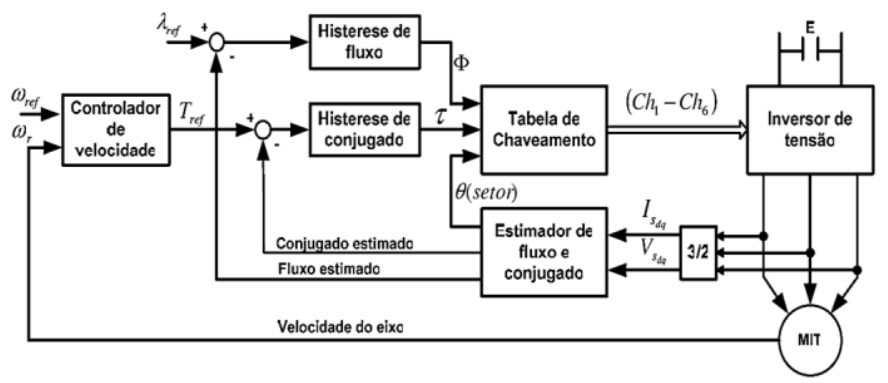

Fig. 1. Diagrama de blocos resumindo o funcionamento do DTC tradicional com malha de controle de velocidade, retirado de Tavares, 2007.

A lógica de chaveamento do DTC é responsável por determinar o vetor de tensão ideal para manter as características de funcionamento da máquina dentro dos padrões preestabelecidos. Ao todo são oito possíveis vetores de chaveamento, sendo seis com amplitude diferente de zero e dois vetores nulos, que são selecionados em função dos erros entre os valores de referência e os valores estimados de torque eletromagnético e fluxo.

Para a determinação do erro de fluxo é utilizado um comparador de histerese de dois níveis. A histerese pode ser modelada simplificadamente da seguinte forma:

$$
\left\{\begin{array}{l}
\mathrm{d} \lambda=1,\left|\overrightarrow{\lambda_{\mathrm{s}}}\right| \leq\left|\overrightarrow{\lambda_{\text {sref }}}\right|-\Delta \lambda_{\mathrm{s}} \\
\mathrm{d} \lambda=0,\left|\overrightarrow{\lambda_{\mathrm{s}}}\right| \geq\left|\overrightarrow{\lambda_{\text {sref }}}\right|+\Delta \lambda_{\mathrm{s}}
\end{array}\right.
$$

Onde: $\lambda_{\text {sref }}$ : referência de fluxo do estator, $\Delta \lambda_{\mathrm{s}}:$ largura de histerese de fluxo.

O uso do comparador de histerese permite a existência de dois estados de erro de fluxo. O primeiro estado, $\mathrm{d} \kappa=1$, corresponde aos valores de fluxo estimados inferiores ao valor de referência, enquanto que o segundo estado, $\mathrm{d} K=0$, corresponde as situações restantes. Dessa forma, através da comparação com a referência, a lógica de chaveamento pode aumentar ou diminuir o módulo do fluxo do estator.

A determinação do estado do erro de torque é realizada através de um comparador de histerese de três níveis. O modelo simplificado pode ser demonstrado considerando sentido de rotação horário e anti-horário e levando em conta que as equações não englobam o efeito memória inserido pela histerese do comparador. O equacionamento utilizado para representar o comparador de histerese de três níveis (Eqs. 2 e 3) foi baseado no modelo proposto por Takahashi (1985).

$$
\begin{aligned}
& \underset{\text { Rotação no sentido }}{\text { anti-horário }} \rightarrow \rightarrow\left\{\begin{array}{c}
\mathrm{dT}_{\mathrm{e}}=1,\left|\mathrm{~T}_{\mathrm{e}}\right| \leq \mathrm{T}_{\text {eref }}-\Delta \mathrm{T}_{\mathrm{e}} \\
\mathrm{dT}_{\mathrm{e}}=0, \mathrm{~T}_{\mathrm{e}} \geq \mathrm{T}_{\text {eref }}
\end{array}\right. \\
& \underset{\text { Rotação no sentido }}{\text { horário }} \rightarrow \rightarrow\left\{\begin{array}{c}
\mathrm{dT}_{\mathrm{e}}=-1,\left|\mathrm{~T}_{\mathrm{e}}\right| \geq \mathrm{T}_{\text {eref }}+\Delta \mathrm{T}_{\mathrm{e}} \\
\mathrm{dT}_{\mathrm{e}}=0, \mathrm{~T}_{\mathrm{e}} \leq \mathrm{T}_{\text {eref }}
\end{array}\right.
\end{aligned}
$$


Sendo: $\mathrm{T}_{\text {eref }}$ : referência de torque eletromagnético, $\Delta \mathrm{T}_{\mathrm{e}}$ : largura de histerese de torque.

O comparador de três níveis define três possibilidades de erro: nulo, positivo e negativo, que variam conforme haja necessidade de manter o torque inalterado, incrementá-lo ou decrementá-lo, respectivamente.

Outro parâmetro necessário para o funcionamento do DTC está relacionado ao setor espacial onde se encontra o fluxo do estator. Essa posição é determinada através da divisão do plano $\alpha \beta$ em seis setores de forma que no interior de cada um existe apenas um vetor espacial de tensão. Além disso, o modelo de simulação do motor empregado para ser aplicado na lógica do Controle Direto de Torque utiliza o sistema de referência estacionário coincidindo com a fase $\mathrm{A}$, na qual $\mathrm{w}_{\mathrm{e}}=0$.

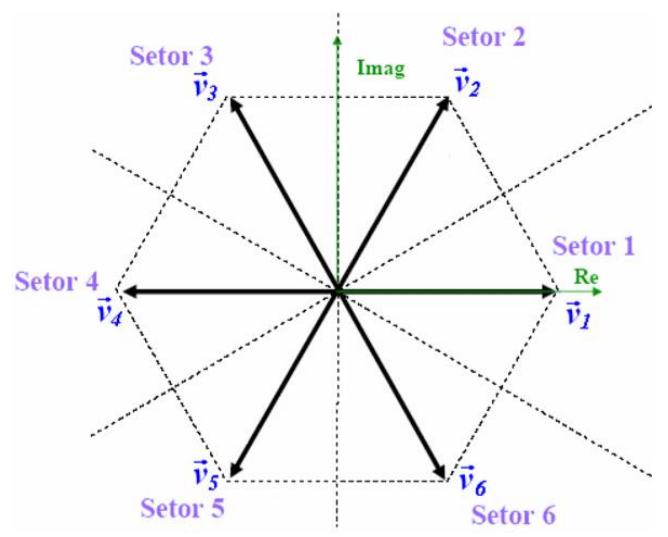

Fig. 2. Distribuição e denominação dos setores espaciais, retirado de Castoldi (2006).

Para o cálculo do setor em que se encontra o fluxo do estator, são utilizadas as equações abaixo, obtidas através de relações trigonométricas, sendo posteriormente aplicadas as desigualdades da tabela 1 .

$$
\cos \left(\theta_{\mathrm{e}}\right)=\frac{\lambda_{\alpha \mathrm{s}}}{\left|\vec{\lambda}_{\mathrm{s}}\right|} \quad ; \operatorname{sen}\left(\theta_{\mathrm{e}}\right)=\frac{\lambda_{\beta \mathrm{s}}}{\left|\vec{\lambda}_{\mathrm{s}}\right|}
$$

Tabela 1. Distribuição dos Setores Espaciais

\begin{tabular}{|c|c|c|}
\hline & $\cos \left(\theta_{\mathrm{e}}\right)$ & $\operatorname{sen}\left(\theta_{\mathrm{e}}\right)$ \\
\hline Setor 1 & $\sqrt{3} / 2<\cos \left(\theta_{\mathrm{e}}\right)$ & - \\
\hline Setor 2 & $0 \leq \cos \left(\theta_{\mathrm{e}}\right) \leq \sqrt{3} / 2$ & $0 \leq \operatorname{sen}\left(\theta_{\mathrm{e}}\right)$ \\
\hline Setor 3 & $-\sqrt{3} / 2 \leq \cos \left(\theta_{\mathrm{e}}\right)<0$ & $0 \leq \operatorname{sen}\left(\theta_{\mathrm{e}}\right)$ \\
\hline Setor 4 & $\cos \left(\theta_{\mathrm{e}}\right)<-\sqrt{3} / 2$ & - \\
\hline Setor 5 & $-\sqrt{3} / 2 \leq \cos \left(\theta_{\mathrm{e}}\right)<0$ & $\operatorname{sen}\left(\theta_{\mathrm{e}}\right)<0$ \\
\hline Setor 6 & $0 \leq \cos \left(\theta_{\mathrm{e}}\right) \leq \sqrt{3} / 2$ & $\operatorname{sen}\left(\theta_{\mathrm{e}}\right)<0$ \\
\hline
\end{tabular}

Diante dos três parâmetros necessários para aplicação da lógica, são utilizadas estratégias de funcionamento que possibilitam a escolha dos vetores de tensão. A estratégia adotada nesse trabalho engloba o uso de relê de histerese de três níveis para o controle do torque, possibilitando chaveamentos com vetores nulos e consequente redução da frequência de chaveamento do inversor.

A lógica de chaveamento adotada utiliza um vetor de tensão que gera um incremento de torque no sentido contrário ao movimento sempre que há necessidade de reduzir o torque elétrico da máquina. Com isso, ela possibilita uma resposta de torque rápida e uma operação nos quatro quadrantes, ocasionando em compensação um chaveamento mais intenso do inversor quando comparada com outras estratégias. A Tabela 2 descreve a lógica de chaveamento utilizada na programação do DTC através do conceito de vetores espaciais, na qual cada vetor corresponde a um estado do conversor de frequência.

\section{Tabela 2 - Lógica de Chaveamento utilizada na programação do DTC}

\begin{tabular}{|c|c|c|c|c|c|c|c|}
\hline \multicolumn{2}{|c|}{} & $\theta(1)$ & $\theta(2)$ & $\theta(3)$ & $\theta(4)$ & $\theta(5)$ & $\theta(6)$ \\
\hline \multirow{4}{*}{$\varphi=1$} & $\Phi=1$ & $\overline{V_{2}}$ & $\overline{V_{3}}$ & $\overline{V_{4}}$ & $\overline{V_{5}}$ & $\overline{V_{6}}$ & $\overline{V_{1}}$ \\
\cline { 2 - 9 } & $\Phi=0$ & $\overline{V_{7}}$ & $\overline{V_{0}}$ & $\overline{V_{7}}$ & $\overline{V_{0}}$ & $\overline{V_{7}}$ & $\overline{V_{0}}$ \\
\cline { 2 - 9 } & $\Phi=-1$ & $\overline{V_{6}}$ & $\overline{V_{1}}$ & $\overline{V_{2}}$ & $\overline{V_{3}}$ & $\overline{V_{4}}$ & $\overline{V_{5}}$ \\
\hline \multirow{4}{*}{$\varphi=0$} & $\Phi=1$ & $\overline{V_{3}}$ & $\overline{V_{4}}$ & $\overline{V_{5}}$ & $\overline{V_{6}}$ & $\overline{V_{1}}$ & $\overline{V_{2}}$ \\
\cline { 2 - 9 } & $\Phi=0$ & $\overline{V_{0}}$ & $\overline{V_{7}}$ & $\overline{V_{0}}$ & $\overline{V_{7}}$ & $\overline{V_{0}}$ & $\bar{V}_{7}$ \\
\cline { 2 - 9 } & $\Phi=-1$ & $\overline{V_{5}}$ & $\overline{V_{6}}$ & $\overline{V_{1}}$ & $\overline{V_{2}}$ & $\overline{V_{3}}$ & $\bar{V}_{4}$ \\
\hline
\end{tabular}

\subsection{Estimador de Torque Eletromagnético}

O método de estimação do torque eletromagnético inserido na malha de controle em substituição ao torquímetro foi analisado por Mendes (2015). O "Método do KTorque" utiliza as tensões e correntes de alimentação do motor para a estimação do torque. Essa técnica trabalha com as equações referenciadas aos eixos dq, de forma que o eixo em quadratura coincida com a fase " $A$ " da tensão de alimentação e possui velocidade angular síncrona com a rede.

A partir de estudos analíticos dos resultados da simulação do estimador, conclui-se que:

$$
\mathrm{i}_{\mathrm{qs}}^{\mathrm{e}} \approx-\mathrm{i}_{\mathrm{qr}}^{\mathrm{e}} ; \mathrm{i}_{\mathrm{dr}}^{\mathrm{e}} \approx 0 ; \mathrm{i}_{\mathrm{ds}}^{\mathrm{e}} \approx \text { constante }
$$

A equação de torque do modelo dinâmico pode ser reproduzida substituindo os enlaces de fluxos no estator para obter uma nova expressão de torque eletromagnético baseada apenas nas variáveis de corrente nos eixos dq, como pode ser observado na Equação 5.

$$
\mathrm{T}_{\mathrm{e}}=\frac{3}{2} \frac{\mathrm{P}}{2} \mathrm{~L}_{\mathrm{m}}\left(\mathrm{i}_{\mathrm{qs}} \mathrm{i}_{\mathrm{dr}}-\mathrm{i}_{\mathrm{ds}} \mathrm{i}_{\mathrm{qr}}\right)
$$

Com isso, a partir das três conclusões anteriores, a Equação 5 pode então ser reescrita da seguinte forma:

$$
\mathrm{T}_{\mathrm{e}}=\mathrm{k}_{\text {torque }} \mathrm{i}_{\mathrm{qs}}^{\mathrm{e}}
$$

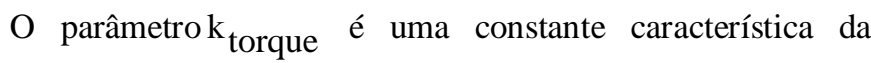
máquina, assumida invariável ao longo de toda faixa de operação. O levantamento dessa constante é realizado através dos dados informados pela placa de identificação do motor e 
depende exclusivamente da corrente de estator, do fator de potência e do torque nas condições nominais de operação.

Calcula-se o valor nominal de $\mathrm{i}_{\mathrm{q}}^{\mathrm{e}}$ através da Equação 7, obtida pela transformação de coordenadas para os eixos dq considerando o referencial fixo no estator. Dessa forma, o torque eletromagnético pode ser estimado considerando uma grande faixa de operação. A Equação 8 permite chegar ao valor atribuído a constante $K_{\text {torque, tendo em conta os valores }}$ nominais de corrente e torque.

$$
\begin{gathered}
\mathrm{i}_{\mathrm{qsn}}^{\mathrm{e}}=\sqrt{2} \mathrm{I}_{\mathrm{sn}} \cos \theta_{\mathrm{n}} \\
\mathrm{K}_{\text {torque }}=\frac{\mathrm{T}_{\mathrm{en}}}{\mathrm{i}_{\mathrm{qsn}}^{\mathrm{e}}}
\end{gathered}
$$

O torque da máquina é uma grandeza não linear, porém o método assume uma linearidade durante a faixa de operação. Os estudos apresentados por Mendes (2015) mostraram que o estimador possui resultados semelhantes ao calculado pela equação dinâmica do modelo do MIT. Os valores obtidos pelo método do Ktorque também foram comparados com a leitura de um torquímetro, demonstrando a robustez da técnica.

\section{RESULTADOS}

Os ajustes do controlador PID na malha do DTC foram realizados considerando os princípios do controle em cascata clássico onde as malhas mais internas são suficientemente mais rápidas que as malhas mais externas Além disso, a rigidez dinâmica e a robustez foram preservados de forma a evitar que perturbações de cargas e erros de estimação de parâmetros da planta não levem a erros grandes no rastreamento da trajetória.

Os ganhos proporcional e integral foram calculados através do levantamento da função de transferência da malha w/Tc' juntamente com o polinômio característico do sistema, na qual os polos possuem apenas a componente real. As Equações 9 e 10 correspondem ao cálculo das constantes proporcional e integral. A constante derivativa foi calculada em função da constante integral, utilizando um fator de multiplicação conforme a Equação 11.

$$
\begin{gathered}
\text { Kp }=2 \pi J\left(B_{\text {Wint }}+B_{\text {Wprop }}\right)-B \\
\text { Kint }=4 \pi^{2} \mathrm{~J}\left({ }^{B_{\text {Wint }}}{ }^{B_{\text {Wprop }}}\right) \\
\text { Kder }=10^{-4} \text { Kint }
\end{gathered}
$$

Sendo que $\mathrm{B}_{\mathrm{Wprop}}$ corresponde a faixa de passagem da banda proporcional e $\mathrm{B}_{\text {Wint }}$ correspondente a banda integral.

Foi utilizado como referência um sinal de velocidade variável com valores positivos e negativos e com dois estágios diferentes de velocidade (1710 RPM e -850 RPM). Para os resultados gráficos apresentados aqui considerou-se o torque requerido pela carga constante, porém a utilização do estimador aplicado a lógica do DTC também permite o uso de carga com característica linear ou quadrática com a velocidade, apresentando resultados semelhantes nas simulações realizadas. A trajetória de velocidade utilizada é apresentada na Figura 3.

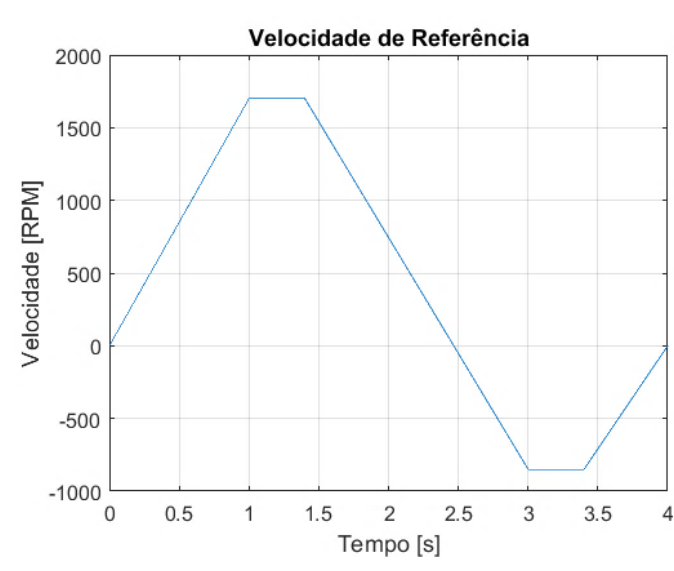

Fig. 3. Característica de velocidade de referência

Foram realizadas simulações envolvendo o uso do estimador inserido da malha do DTC e empregando o torque eletromagnético real, calculado a partir da equação retirada do modelo dinâmico da máquina de indução.

Analisando os dois casos percebe-se que o uso do estimador aplicado a malha produziu um resultado semelhante ao uso do torque eletromagnético real, calculado a partir da equação do modelo dinâmico. A utilização do "Método do KTorque" na malha simplifica a implementação da técnica DTC, pois o método de estimação do torque propõe a utilização de dados contidos na placa de identificação do motor e depende apenas das tensões e correntes de alimentação, que podem ser obtidas de forma não invasiva.

Além disso, a equação dinâmica de torque depende do conhecimento dos enlaces de fluxo do estator que dificultam a implementação prática da lógica DTC. Segundo Rosa (2004) e Lacerda (2016), a obtenção das variáveis de fluxo depende da resolução de integrais e a presença de componentes contínuas nas variáveis coletadas acabam gerando uma divergência no resultado final devido a um erro crescente somado a cada passo de integração. Dessa forma, utilizando o estimador apresentado, evita-se a necessidade de compensação de sinais no algoritmo de tratamento das variáveis e reduz o número de iterações necessários a cada integração.

As Figuras 4 e 5 apresentam o erro de velocidade encontrado utilizando o torque real da máquina e o estimador, respectivamente.

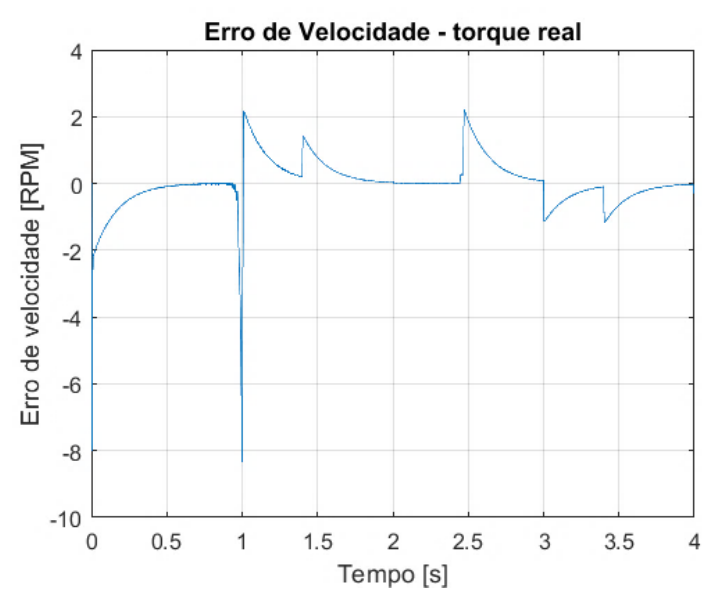

Fig. 4. Erro de velocidade utilizando torque eletromagnético real da máquina 


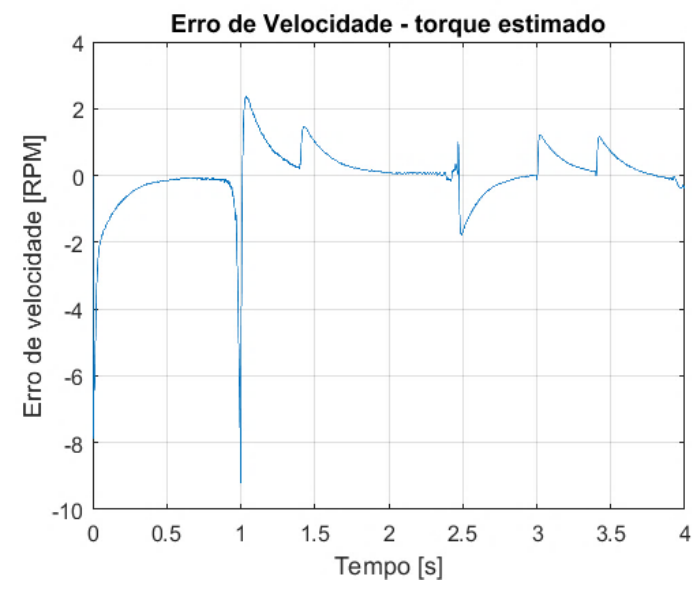

Fig. 5. Erro de velocidade utilizando torque eletromagnético estimado

Observou-se que nas duas situações, o valor absoluto atribuído ao erro de velocidade é o mesmo. Ocorre uma pequena diferenciação no instante equivalente a 2,5 segundos devido a inversão do sentido da caraterística de velocidade da máquina e a forma com que o estimador trabalha essa alteração para ser repassada a lógica de programação do DTC. O valor de torque estimado repassado ao DTC está um período de amostragem atrasado em relação ao valor instantâneo da velocidade de referência, pois a estimação do torque depende do valor da corrente elétrica no mesmo instante de tempo da velocidade.

Utilizando o estimador de torque analisado, o conjugado apresentou oscilações semelhantes ao apresentado pelo torque real, evidenciado pela característica de erro (Figura 8) com valores próximos de 1 N.m. Em ambos os casos, os parâmetros utilizados na malha de controle de velocidade e na lógica do DTC foram determinantes para controlar o ripple desenvolvido no motor.

Além da comparação entre o erro de velocidade e a resposta de torque para as duas situações consideradas, uma outra característica que pode atestar a eficácia do método apresentado é o comportamento da corrente que percorre os enrolamentos do estator. Observou-se que mesmo diante das variações no torque desenvolvido pela máquina e nos momentos de aceleração e reversão de velocidade, a corrente nas três fases esteve dentro dos valores esperados, atingindo valor nominal nas situações de regime permanente. A figura 6 expõe o sinal de corrente obtido para a fase A do estator.

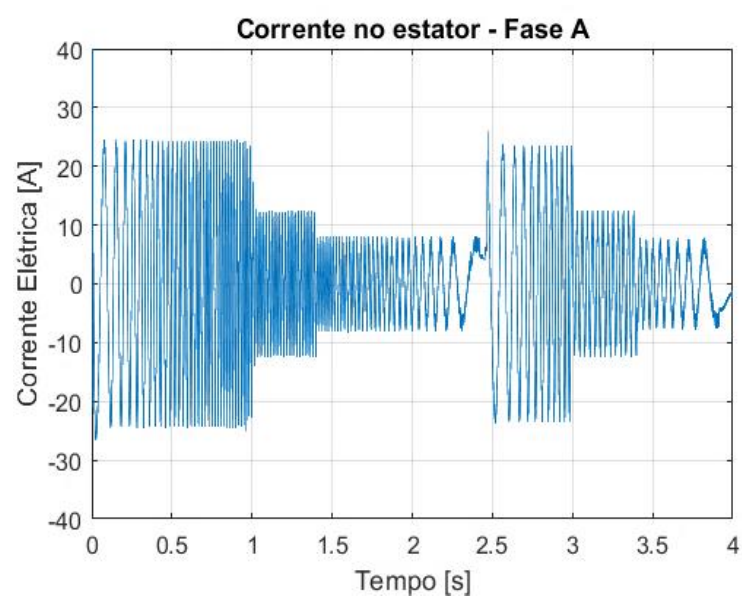

Fig. 6. Corrente de alimentação do estator - Fase A
A figura 7 apresenta o comportamento de toque do MIT utilizando o estimador para compor da malha de controle do DTC.

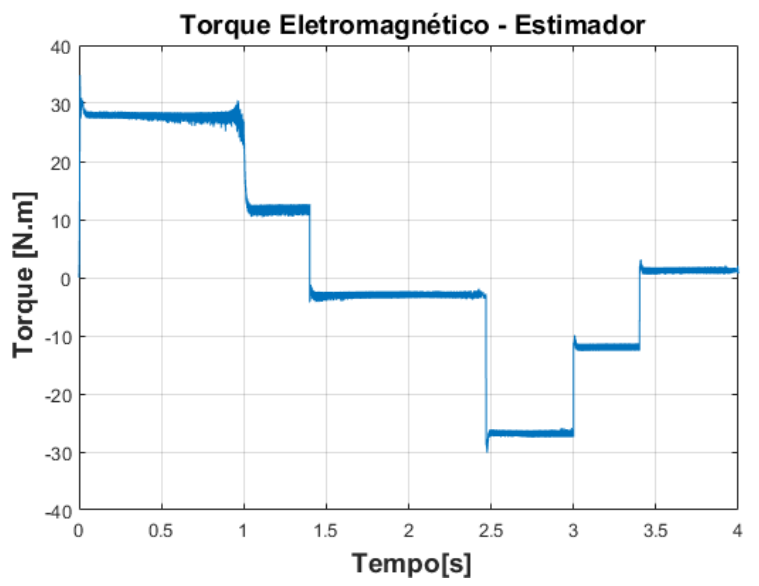

Fig. 7. Torque desenvolvido utilizando o estimador na malha de comando

A figura 8 apresenta o erro obtido para o torque da máquina comparando o comportamento utilizando o torque real e o torque obtido pelo estimador na malha de controle. Para essas situações foi utilizado um fluxo de referência constante de 0.44 $\mathrm{Wb}$ e o torque de referência variável de acordo com a resposta do controlador PID alimentado pelo erro de velocidade.

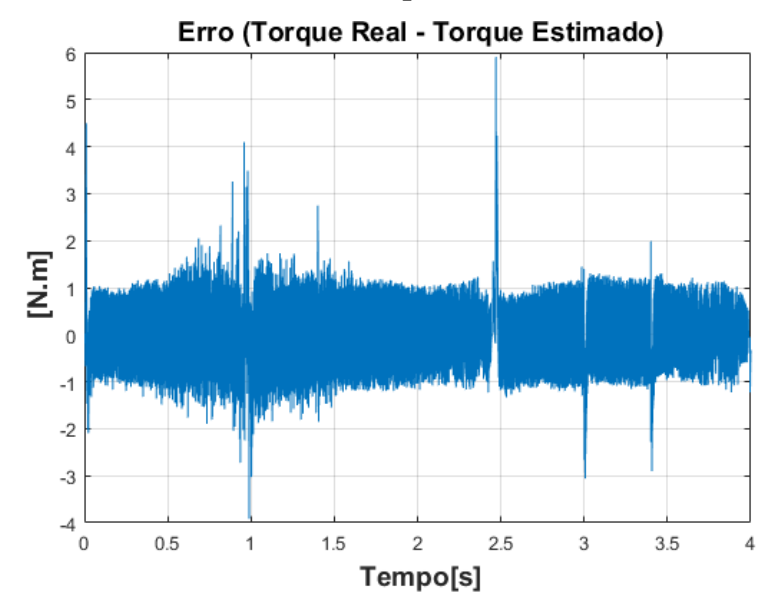

Fig. 8. Erro de torque comparando o toque real e o estimado na malha de comando do DTC

Durante a análise dos resultados apresentados pela inserção do "Método do KTorque" na técnica DTC, o comportamento de torque desenvolvido pela máquina apresentou grandes variações em virtude da escolha do ganho do controlador derivativo. A escolha desse valor levou em consideração o valor da constante integral calculada pela malha.

Os valores dos ganhos integral e proporcional foram calculados a partir do método proposto por Lorenz, Lipo e Novotny (1994). Nesse trabalho, definiu-se os ganhos para atender três características: faixa de passagem, rejeição a perturbação (ou rigidez dinâmica) e robustez do sistema (ou invariância do comportamento dinâmico frente a erros de parâmetros na planta).

Foram estudados três casos: ausência do termo derivativo, constante $\mathrm{Kd}_{1}$ e constante $\mathrm{Kd}_{2}$, sendo $\mathrm{Kd}_{1}>\mathrm{Kd}_{2}$. As Figuras 911 apresentam as respostas do torque para esses três valores das constantes do termo derivativo. 


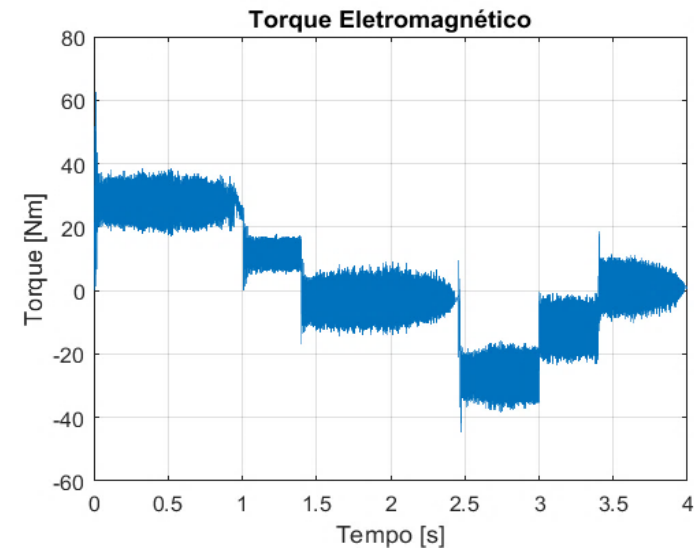

Fig. 9. Torque eletromagnético desenvolvido pelo motor inserido em uma malha com ausência do termo derivativo (Controlador PI)

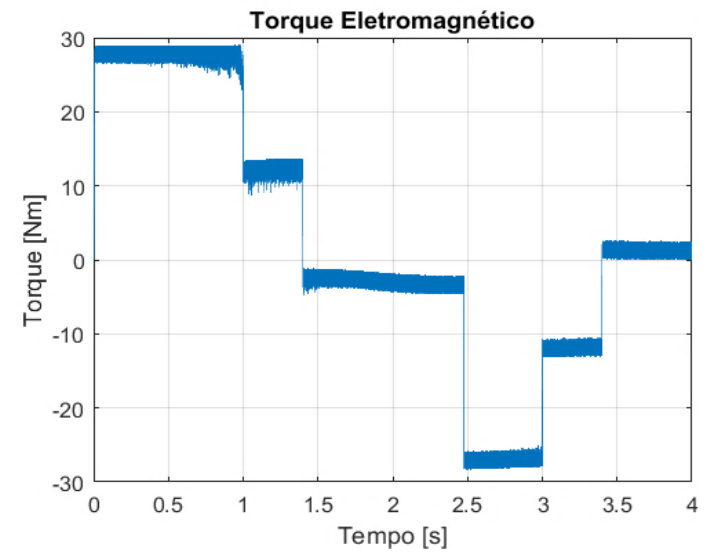

Fig. 10. Torque eletromagnético desenvolvido pelo motor inseridoControlador PID com constante $\mathrm{Kd}_{1}$

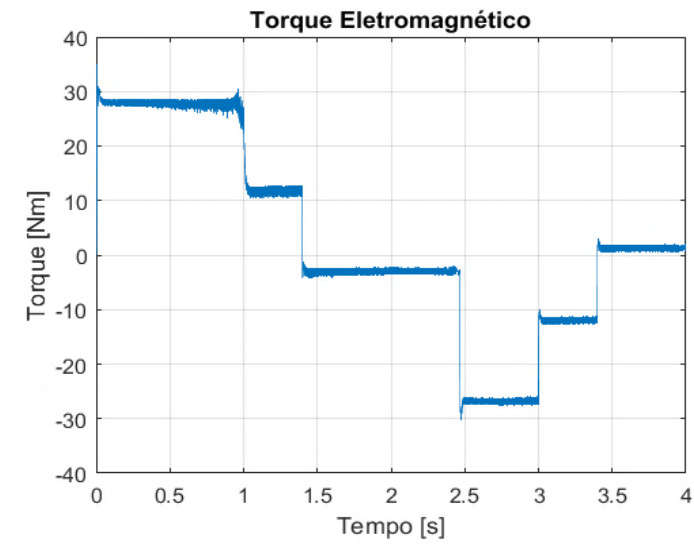

Fig. 11. Torque eletromagnético desenvolvido pelo motor inseridoControlador PID com constante $\mathrm{Kd}_{2}$

\section{CONCLUSÕES}

O método de estimação de torque apresentado utilizando os dados da placa de identificação e a corrente do eixo em quadratura obteve resultados semelhantes ao torque real. Além do mais, a simplicidade de aplicação do estimador faz com que a técnica possa ser diretamente aplicada a malha de controle vetorial do DTC.

A proximidade de resultados do erro absoluto de velocidade da malha e das oscilações na resposta de torque atestam a confiabilidade do estimador durante sua aplicação. Notou-se que o método apresenta grandes variações de resposta na malha do Controle Direto de Torque em decorrência da escolha correta dos parâmetros dos comparadores de histerese e dos ganhos inerentes ao controlador PID, responsável pela malha de velocidade.

A estimação do torque, abordada nesse trabalho, surge como uma alternativa a umas das principais desvantagens da aplicação da técnica do DTC, que é a necessidade de estimação dos parâmetros como o torque eletromagnético.

\section{AGRADECIMENTOS}

Os autores agradecem à CAPES/CNPq/FAPEMIG/Inerge e à UFSJ pelos auxílios financeiros recebidos.

\section{REFERÊNCIAS}

Blaschke, Felix. "The Principle of Field Orientation as Applied to the New Transvector Closed-Loop Control System for Rotating Field Machines", Siemes Review, 1972.

Castoldi, M. F. (2006). Protótipo virtual da estratégia DTC aplicada a motores de indução usando linguagem VHDL. Master's Dissertation, Escola de Engenharia de São Carlos, University of São Paulo, São Carlos.

Krause, P. C., Wasynczuk, O. and Sudhof, S. D. (2002). Analysis of Electric Machinery and Drive Systems, Academic Press - New Jersey.IEEE Transactions on Power Systems, Vol. 9, No. 4, pp. 1942- 1948.

Lacerda, G.A.M. Estimação de Torque de Motor de Indução Trifásico utilizando Instrumentos comuns em indústrias. Programa de Pós-Graduação em Engenharia Elétrica CEFET/UFSJ. UFSJ, São João del-Rei, MG. 2016. Dissertação de mestrado.

Mendes, L.F.S. Proposta de um Método para Estimação de Torque em Motores de Indução Trifásicos e aplicação na Detecção de Falhas. Programa de Pós-Graduação em Engenharia Elétrica CEFET/UFSJ. UFSJ, São João delRei, MG. 2015. Dissertação de mestrado.

Novotny, D.W; Lipo, T. A. "Vector control and dynamics of AC drives" Oxford: Clarendon, 1996. 440 p.

Malla, S. G. "A review on Direct Torque Control (DTC) of induction motor: with applications of fuzzy," 2016 International Conference on Electrical, Electronics, and Optimization Techniques (ICEEOT), Chennai, 2016, pp. 4557-4567.

Takahashi, I., Noguchi, T., “A New Quick Response and HighEfficiency Control Strategy of an Induction Motor", IEEE IAS Ann. Mtg., 1985, P. 496-502.

Tavares, T. S., Study of the direct torque control technique applied to cage induction machines. 2007. $99 \mathrm{f}$. Dissertação (Mestrado em Engenharias) - Universidade Federal de Uberlândia, Uberlândia, 2007.

Tazerart, F., Mokrani, Z., Rekioua, D., Rekioua, T., Direct torque control implementation with losses minimization of induction motor for electric vehicle applications with high operating life of the battery, International Journal of Hydrogen Energy, Volume 40, Issue 39, 2015, Pages 13827-13838 